\title{
CONTINUITY OF THE KOBAYASHI METRIC IN DEFORMATIONS AND FOR ALGEBRAIC MANIFOLDS OF GENERAL TYPE ${ }^{1}$
}

BY MARCUS W. WRIGHT

Communicated by J. A. Wolf, July 26, 1976

Let $M$ be a complex manifold and $T M$ the holomorphic tangent bundle of $M$. The disc of radius $r$ in $\mathrm{C}$ will be denoted by $\Delta(r)$, and $\Delta$ will stand for $\Delta(1)$. The Kobayashi pseudo-distance $d_{M}$ and its infinitesimal pseudo-metric $F_{M}$ are defined as follows:

(i) If $p, q \in M$, then

$$
d_{M}(p, q)=\inf _{\left\{a_{i}\right\} \subset \Delta} \frac{1}{2} \sum_{i} \log \frac{1+\left|a_{i}\right|}{1-\left|a_{i}\right|}
$$

where the infimum is over all finite sets $\left\{a_{i}\right\} \subset \Delta$ such that there exist $n$ analytic mappings $f_{i}: \Delta \rightarrow M$ for which $f_{1}(0)=p, f_{i}\left(a_{i}\right)=f_{i+1}(0)$ for $i=1, n-1$, and $f_{n}\left(a_{n}\right)=q$.

(ii) If $\langle x, \xi\rangle \in T M$, then $F_{M}(x, \xi)=\inf 1 / R$ where the infimum is over all $R$ such that there exists an analytic $f: \Delta(R) \rightarrow M$ with $f_{x}\left(0, \partial /\left.\partial z\right|_{0}\right)=$ $\langle x, \xi\rangle$.

Royden has shown [5] that $d_{M}(p, q)=\inf _{\sigma} \int_{\sigma} F(\sigma, \dot{\sigma})$ where the infimum is over all piecewise smooth curves from $p$ to $q$.

The manifold $M$ is said to hyperbolic if $d_{M}(p, q) \neq 0$ whenever $p \neq q$.

A deformation of $M$ is specified by giving an analytic space $S \subset \mathbf{C}^{k}$ and a family of integrable almost complex structures $\left\{\varphi_{s} \mid s \in S\right\}$ on $M$ such that $\varphi_{0}=$ 0 for some point $o \in S$; each $\varphi_{s}$ is therefore a $C^{\infty} T M$-valued $(0,1)$ form on $M$, satisfying $\bar{\partial} \varphi_{s}-\left[\varphi_{s}, \varphi_{s}\right] / 2=0$. See [2] for details. Using $\varphi_{s}$, we can construct a bundle isomorphism $\Phi_{s}: T M \longrightarrow T M_{s}$, where $T M_{s}$ is the holomorphic tangent bundle for the complex structure given by $\varphi_{s}$. Set $F_{M_{s}}=F_{s}$. Assume that $o=0$, the origin in $\mathbf{C}^{k}$.

Theorem A. Given $\langle x, \xi\rangle \in T M$ and $\epsilon>0$, there exists $a \delta>0$ such that if $|s|<\delta$ then $F_{s}(y, \eta) \leqslant F_{o}(x, \xi)+\epsilon\|\xi\|$ for all $\langle y, \eta\rangle$ in a neighborhood of $\left\langle x, \Phi_{s} \xi\right\rangle$ in $T M_{s}$. (Here $\|\xi\|$ is the norm provided by a coordinate system.)

This basic upper semicontinuity result can be improved if $F_{M}$ is known to

AMS (MOS) subject classifications (1970). Primary 32H15, 32H20, 32G17, $32 \mathrm{G} 05$.

1 This work supported in part by NSF Grant MPS-75-05270. 
be continuous on $T M$; e.g., if $F_{M}$ is continuous and $M$ is compact, the $\delta$ can be chosen to be independent of $\langle x, \xi\rangle$.

THEOREM B. If $M$ is compact and hyperbolic, then $F_{s}(y, \eta)$ is continuous on $\bigcup_{s \in U} T M_{s}$ and $d_{s}(p, q)$ is continuous on $U \times M \times M$ for $U$ any sufficiently small neighborhood of $o \in S$.

This theorem follows from Theorem A and the result of R. Brody [1] that $F_{s}$ is lower semicontinuous in $s$ for $s$ sufficiently close to 0 when $M$ is hyperbolic.

Using Theorem B and the Kuranishi theory of versal deformations [2], we obtain the following result about moduli of compact hyperbolic manifolds. See [3] for a similar result for manifolds with ample canonical bundle.

Theorem C. Let $M$ be a compact hyperbolic manifold and let $\mathfrak{M}$ denote the collection of isomorphism classes of hyperbolic complex structures on the underlying differentiable manifold of $M$. Then $\mathfrak{M}$ has the structure of a Hausdorff complex space such that if $\left\{M_{s}\right\}_{s \in S}$ is any family of hyperbolic complex structures on $M$, then the map sending $s$ to the isomorphism class of $M_{s}$ is a morphism from $S$ to $\Re$.

Examples of Royden (unpublished) show that $F_{M}$ is not always lower semicontinuous on $M$.

Definition. A projective algebraic manifold $M$ is said to be of general type if

$$
\limsup _{m \rightarrow+\infty} \frac{1}{m^{n}} H^{0}\left(M, O\left(K^{m}\right)\right)>0
$$

Here $K$ denotes the canonical bundle and $\operatorname{dim}_{\mathrm{C}} M=n$. Let $\eta$ denote the KobayashiEisenman pseudo-volume for $M$ [4] .

THEOREM D. If $M$ is compact algebraic and there exist sections $S_{0}, \ldots, S_{k}$ of $H^{0}\left(M, O\left(K^{m}\right)\right)$ which provide a projective embedding of $M$ such that $S_{i} \bar{S}_{i} / \eta$ is bounded for every $i=0, \ldots, k$, then $F_{M}$ is continuous on $T M$.

COROLLARY. If $M$ is projective algebraic of general type then $F_{M}$ is continuous on TM.

Proofs and details of the above will appear in [6].

\section{REFERENCES}

1. R. Brody, Intrinsic metrics and measures on compact complex manifolds, Thesis, Harvard Univ., 1975.

2. M. Kuranishi, Deformations of compact complex manifolds, Séminaire de Mathématiques Supérieures, No. 39 (Été 1969), Univ. of Montréal Press, Montréal, Quebec, 1971. MR 50 \# 7588. 
3. M. S. Narasimhan and R. R. Simha, Manifolds with ample canonical class, Invent. Math. 5 (1968), 120-128. MR 38 \# 5253.

4. D. A. Pelles (formerly Eisenman), Holomorphic maps which preserve intrinsic measure, J. Math. 97 (1975), 1-15. MR 51 \# 3542.

5. H. L. Royden, Remarks on the Kobayashi metric, Several Complex Variables. II (Proc. Internat. Conf., Univ. of Maryland 1970), Lecture Notes in Math., vol. 185, SpringerVerlag, Berlin and New York, 1971, pp. 125-137. MR 46 \# 3826.

6. M. W. Wright, The Kobayashi pseudo-metric on algebraic manifolds of general type and in deformations of complex manifolds, Trans. Amer. Math. Soc. (to appear).

DEPARTMENT OF MATHEMATICS, UNIVERSITY OF KENTUCKY, LEXINGTON, KENTUCKY 40506 